Original article

\title{
Clinical manifestations, histopathological changes and quality of life in patients with advanced knee osteoarthritis caused by age, trauma, obesity and their combination
}

\author{
Kristina V. Korochina, Tatyana V. Chernysheva, Irina E. Korochina \\ Orenburg State Medical University, Orenburg, Russia
}

Received 3 December 2020, Revised 18 May 2021, Accepted 27 July 2021

(C) 2020, Russian Open Medical Journal

\begin{abstract}
Objective - to reveal and analyze clinical characteristics, knee joint histopathology, and quality of life in patients at late stages of knee osteoarthritis (OA) triggered by age, trauma, metabolic syndrome, or their combination.

Material and methods - We studied 120 subjects with knee OA (sensu Altman R.D., 1991) of Kellgren-Lawrence Grades 3-4. They were distributed among 4 groups (30 participants in each) based on the presence of age-related, post-traumatic, metabolic, or combined phenotypes. Clinical examination of patients with pain and their functional status evaluation (via Visual Analogue Scale, WOMAC, Lequesne index), quality of life assessment (MOS SF-36 questionnaire), along with histopathological study of medial tibial plateau cartilage and synovial membrane, were caried out, followed by statistical data processing.

Results - Age-related OA phenotype was characterized by the latest clinical onset [59.5 (54-68) years of age] with the largest average patient age [72.5 (63-77) years], moderate to severe pain and knee dysfunction [total WOMAC score of 160 (127-190) points and Lequesne index of 20 (8-21) points], severe degenerative cartilage lesions [8.5 (6-10) points sensu Mankin] with high-grade synovitis [5 (3-8) points sensu Krenn]. Post-traumatic OA phenotype was distinguished by the lowest pain, stiffness and knee functional limitations [total WOMAC score of 129 (100-166), Lequesne index of 15 (14-19)], the highest quality of life in patients [physical component summary of 34.1 (30.536.1) points, mental component summary of 40.4 (32.9-43.8) points] against the background of local severe cartilage lesions [8 (6-8) sensu Mankin] with reparative pattern and synovial fibrosis. For metabolic OA phenotype, the typical traits included female-biased sex ratio (87\%), high prevalence of clinical synovitis (77\%), severe pain and functional knee disorders [total WOMAC score of 188 (162-207) points, Lequesne index of 20 (19-23) points], the worst quality of life [physical component summary of 28.0 (24.3-31.9) points, mental component summary of 30.9 (26.9-35.9) points], vascular invasion of cartilage, and high-grade synovitis [4 (3-5) points sensu Krenn]. Combined OA phenotype was characterized by variable clinical and histopathological features.

Conclusion - Comprehensive comparative clinical and morphological analysis of late-stage knee OA of various origin was completed, and age-related, post-traumatic, metabolic and combined OA phenotypes were studied. The methodological basis for differential approach to treating different categories of OA patients was developed.
\end{abstract}

Keywords: knee osteoarthritis, phenotype, risk factor, WOMAC, quality of life, histopathology.

Cite as Korochina KV, Chernysheva TV, Korochina IE. Clinical manifestations, histopathological changes and quality of life in patients with advanced knee osteoarthritis caused by age, trauma, obesity and their combination. Russian Open Medical Journal 2021; 10 : e0312.

Correspondence to Kristina V. Korochina. Address: 23/1 Novaya St., Apt. 37., Orenburg 460050, Russia. Phone: +79878470879. E-mail: kris kor@inbox.ru.

\section{Introduction}

The relevance of studying osteoarthritis (OA) is justified by its extremely high and progressively increasing prevalence worldwide. At the same time, no convincing improvement in its treatment has yet been noted, there is no actual disease-modifying therapy, and used symptomatic medications are not enough effective. In fact, joint ailments, not meeting clear criteria for some other articular nosology, are subjectively attributed to OA.

$\mathrm{OA}$ is considered a disorder of movable joints characterized by cell stress and extracellular matrix degradation initiated by microand macrotrauma activating maladaptive repair responses including pro-inflammatory pathways of innate immunity [1]. First, it manifests itself as a molecular derangement and abnormal joint tissue metabolism with subsequent structural and physiologic derangements, developing into clinical manifestations and outcomes.

Disappointing statistics indicates that since the mid-20th century, the OA prevalence has doubled, and now 81 million OA patients are registered in five developed countries (Germany, Italy, France, Great Britain, Spain), whereas 380 million OA patients are known in Russia, Brazil, India and China [2]. According to the prognosis, by 2050, 130 million people would suffer from OA worldwide, of whom 40 million could be severely disabled by the disease [3].

Clinical and pathogenic heterogeneity of OA actualizes the issue of its stratification because of the search for optimal patient treatment strategy. It has been proposed that $\mathrm{OA}$ is a syndrome comprised of multiple distinct phenotypes rather than a single disease [4]. 


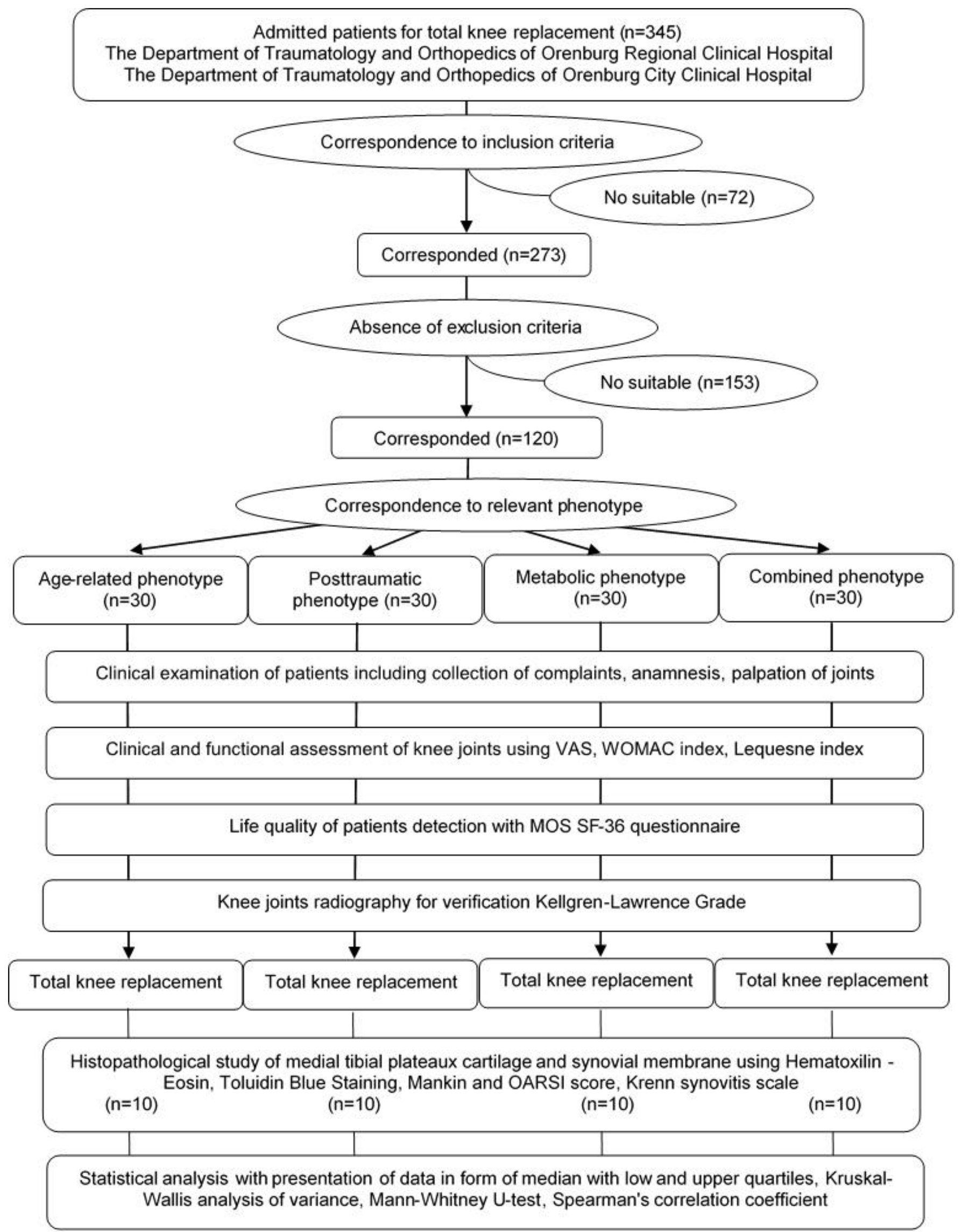

Figure 1. Study design. 
This heterogeneity may be one of the major factors associated with the complexity of OA and with the difficulties to identify 'onesize-fits-all' therapeutic strategies [5]. The concepts of OA heterogeneity and OA phenotypes are rapidly gaining acceptance, and a growing number of researchers, initiatives and studies focus on this topic [6].

For a long time, in the scientific world, there was a lack of unified understanding of what the $O A$ phenotype should represent. This led to using different classification criteria and abundance of $\mathrm{OA}$ varieties, so that it became impossible to evaluate their hierarchical place and give them some deserved preference in terms of their practical use. The phenotype was intuitively understood as a perceptible outcome of combined impact of internal and external factors - e.g., observable properties of a living organism produced by the interactions among the genotype and the environment [7]; or composite observable characteristics of an individual resulting from genetic and environmental factors acting cumulatively [5]. As there was no official definition of the phenotype, scientists often understood various characteristics of the disease and even identified 'biochemical' [8] and 'metabolic' [9] phenotypes; at the same time, the clinical variants that most fully matched this concept were often modestly called 'subtypes' [10], 'subgroups' [11] or 'profiles' [12]. The proposed OA phenotypes should be recognizable in comprehensive clinical practice; they should assist the practitioner in $\mathrm{OA}$ prevention or in prescribing the personalized therapy. In epidemiological scientific research, the established phenotypes should serve a basis for forming the homogeneous cohort of patients in order to study the relationships among OA parameters, assess treatment efficacy, and develop prognostic OA models [13]. Identifying $O A$ phenotypes is an important research priority because it helps better understanding of the pathways and mechanisms that may be involved in each distinct phenotype and target them more effectively using a variety of preventive and treatment strategies [7].

The relevance of OA phenotyping was supported by the Updated Algorithm Recommendation for the Management of Knee Osteoarthritis [14]: four phenotypes (biomechanical, osteoporotic, metabolic and inflammatory) were proposed by the working group of ESCEO (European Society for Clinical and Economic Aspects of Osteoporosis, Osteoarthritis and Musculoskeletal Diseases) based on the OA etiopathogenesis [15]. The document emphasized that future research efforts should focus on the identification of phenotypes in OA patients.

Consistently growing interest to the research on OA phenotyping shifted the focus of scientists' attention to finding consensus in its definition. An important event in field of rheumatology took place in 2020, and for the first time in history, OA phenotype has acquired its official definition [16]. It was defined as, "... subtype of $O A$ that share distinct underlying pathobiological and pain mechanisms and their structural and functional consequences... The potentially identified phenotype should differ from others in terms of clinically relevant diseasedriving factors and/or outcomes." It became obvious that phenotypes should differ from each other not only in clinical characteristics, but at each stage of disease development: from its onset through exclusive pathogenic pathways and structural reorganization to specific manifestations and outcomes.

It seems unambiguous that certain clinical manifestations of the disease should be formed on morphological basis; however, structural damage phenotypes were less often investigated, compared with the clinical (pain) phenotypes [17]. Hence, the histopathological studies of OA phenotypes seem quite relevant. Earlier experimental study [18] revealed the relationship between initiating $\mathrm{OA}$ mechanisms and structural joint tissues progression in animal models, imitating the development of different human OA phenotypes.

In this regard, special attention should be paid to the integrated approach to investigating OA phenotypes. There is a need to effectively combine parameters that represent different fields (e.g., imaging + clinical data + biochemical markers), that could be relevant for OA phenotyping $[6,16]$. Therefore, comprehensive comparative studies of OA phenotypes involving pathogenetic, structural, and clinical aspects of the disease seem especially relevant. Contemporary top-down phenotyping approach was described in [19], presumably improving our knowledge of OA phenotypes, specified based upon known risk factors or imaging data.

Although, merely defining OA phenotypes in terms of risk factors seems too simplistic [5], their separate contributions in all aspects of OA developing are important to investigate for further studies of patients with more than one risk factor and overlapped pathobiological pathways. Among numerous risk factors, age, joint trauma and obesity are very common in actual clinical practice.

The objective of our study was to identify and analyze clinical characteristics, knee joint histopathology, and quality of life in patients at late stages of knee OA caused by different factors: agerelated, post-traumatic, metabolic, and the combination of all of the above.

\section{Material and methods \\ Study design}

The study was performed in accordance with ethical standards specified in the 1964 Declaration of Helsinki and its later amendments, and approved by the Ethics Committee of Orenburg State Medical University. Of those referred to the Departments of Traumatology and Orthopedics of Orenburg Regional Clinical Hospital and Orenburg City Clinical Hospital for total knee replacement, 120 subjects became the participants of our study.

The inclusion criteria for patients were: (1) presence of knee osteoarthritis sensu Altman R.D. (1991) [20]; (2) Kellgren-Lawrence OA grades 3-4 [21]; and (3) signed voluntary informed consent of patients. Taking into account previous proposed classifications and contribution of the most common OA risk factors, four phenotypes of the disease were studied: age-related, post-traumatic, metabolic, and combined OA. To achieve the pathogenic homogeneity within the groups, an influence of other risk factors was minimized, so that the exclusion criteria were: (1) occupational and (2) professional OA risk factors, (3) systematic sports overload, as well as (4) other rheumatological and autoimmune diseases, (5) knee damage of other (than OA) causes, and (6) a patient's refusal from participation.

We examined age-related OA phenotype in patients over 50 years old in absence of obesity, metabolic syndrome and joint injury. Post-traumatic phenotype was diagnosed in cases of a preceding joint injury followed by knee OA manifestations, confirmed by ultrasonography or MRI in the form of damage to ligaments, menisci or other traumatic changes. Metabolic OA phenotype was evaluated in participants with obesity (body mass index [BMI] above $30 \mathrm{~m} / \mathrm{kg}^{2}$ ) and / or metabolic syndrome [sensu International 
Diabetes Federation (IDF) criteria, 2006] but without previous joint trauma. Combined OA presumed all of above-mentioned risk factors. All patients were older 50 years. Each group consisted of 30 people, i.e., minimum required sample size to reveal the statistically significant differences between groups. All patients underwent clinical examination, clinical and functional assessment of knee joints, life quality detection, radiography, as well as histopathological study of articular structures (for every third participant in the group) with statistical processing of data obtained (Figure 1).

\section{Clinical examination}

Clinical examination of patients included the examination of OA complaints, anamneses, routine laboratory analyses, additional obesity parameters assessment (waist and hip circumferences, visceral fat percentage using fat analyzer OMRON BF-306). All patients underwent filling the Western Ontario and McMaster
Universities (WOMAC) questionnaire [22], as well as Lequesne index [23] and Visual Analogue Scale (VAS). WOMAC index consisted of subscales Pain (5 items), Stiffness (2 items) and Functional limitations (17 items) with every item score range 0-10 (maximum 240 points for the total of 24 questions). The Lequesne algofunctional index evaluated OA severity based on sections Pain / Discomfort, Maximum walked distance and Daily routine activities with a maximum index score of 24 points. VAS ranged from 0 (no pain) to 10 (unbearable pain) points.

\section{Quality of life in patients}

The quality of life in patients was assessed by MOS-SF 36 questionnaire comprising 36 questions, grouped into 8 scales (physical functioning, role physical, bodily pain, general health, vitality, social functioning, role emotional, mental health), which ultimately formed two indicators: the physical component summary and mental component summary [24].

Table 1. The results of clinical examination, histopathological study and quality of life assessment in patients with different knee osteoarthritis phenotypes

\begin{tabular}{|c|c|c|c|c|c|}
\hline & $\begin{array}{c}\text { Age-related } O A \\
\text { phenotype }\end{array}$ & $\begin{array}{c}\text { Post-traumatic } O A \\
\text { phenotype }\end{array}$ & Metabolic OA phenotype & Combined OA phenotype & $\begin{array}{c}\text { p-value } \\
\text { (Kruskal-Wallis } \\
\text { analysis of } \\
\text { variance) } \\
\end{array}$ \\
\hline \multicolumn{6}{|c|}{ Clinical characteristics of patients } \\
\hline Male/female (n, \%) & $12(40) / 18(60)$ & $10(33) / 20(67)$ & $4(13) / 26(87) * p=0.019$ & $4(13) / 26(87) * p=0.019$ & 0.020 \\
\hline Age (years) & $72.5(63-77)$ & $62.5(59-64) * p<0.001$ & $63(57-68) * p=0.010$ & $60(56-64) * p=0.020$ & $<0.001$ \\
\hline OA duration (years) & $9.5(6-15)$ & $10(3-12)$ & $12(7-20)$ & $10(7-15)$ & 0.320 \\
\hline Age at onset of OA (years) & $59.5(54-68)$ & $55(36-62) * p=0.028$ & $48(42-58) * p=0.016$ & $49.5(42-56) * p=0.002$ & 0.002 \\
\hline Body mass index $\left(\mathrm{kg} / \mathrm{m}^{2}\right)$ & $28.7(27.4-29.1)$ & $28.1(27.0-28.8)$ & $33.3(31.6-37.3) * p=0.025^{\#} p=0.019$ & $34.1(31.6-38.3) * p=0.015^{\#} p=0.009$ & $<0.001$ \\
\hline Fat analyzer (\%) & $33.6(30.6-39.8)$ & $32.0(25.8-37.4)$ & $44.2(32.0-44.9) * p=0.007^{\#} p=0.004$ & $41.2(39.2-44.3) * p=0.011^{\#} p=0.008$ & $<0.001$ \\
\hline Waist circumference (cm) & $95(90-104)$ & $95(90-100)$ & $107(103-115) * p=0.044^{*} p=0.046$ & $110(103-116) * \mathrm{p}=0.039^{\#} \mathrm{p}=0.041$ & $<0.001$ \\
\hline Hip circumference $(\mathrm{cm})$ & $106(96-110)$ & $110(100-110)$ & $113(110-123) * p=0.050$ & $116(110-123) * p=0.045$ & $<0.001$ \\
\hline \multicolumn{6}{|c|}{ Clinical characteristics of affected knee joints } \\
\hline & Grade 3: 23 (77) & Grade 3: 25 (83) & Grade 3: 18 (60) & Grade 3: 16 (53) & \\
\hline Kellgren-Lawrence grade (n, \%) & Grade 4: 7 (23) & Grade 4: 5 (17) & Grade 4: $12(40)^{\#} p=0.045$ & Grade 4: $14(47)^{\#} p=0.013$ & 0.02 \\
\hline Synovitis (n, \%) & $13(43)$ & $19(63)$ & $23(77) * p=0.008$ & $19(63)$ & 0.066 \\
\hline VAS $(\mathrm{mm})$ & $7(7-8)$ & $7(6-8)$ & $8(7-9)$ & $7(6-8)$ & 0.120 \\
\hline WOMAC, pain (points) & $32(29-39)$ & $24(18-30)$ & $38(29-44) * p=0.001$ & $36(25-38) \# p=0.050$ & 0.002 \\
\hline WOMAC, stiffness (points) & $13(12-15){ }^{\#} p=0.068$ & $8(4-14)$ & $14(12-18) * p=0.002$ & $14(9-16)$ & 0.004 \\
\hline WOMAC, function (points) & $115(87-137) * * p=0.064$ & $97(75-121) * * p<0.001$ & $136(122-150)$ & $105(93-134)^{* *} \mathrm{p}=0.035$ & $<0.001$ \\
\hline WOMAC, total (points) & $160(127-190)^{\#} p=0.057$ & $129(100-166)$ & $188(162-207)^{\#} p<0.001$ & $155(145-179)$ & $<0.001$ \\
\hline Lequesne index (points) & $20(8-21)^{\#} p=0.004$ & $15(14-19)$ & $20(19-23)^{\#} p=0.003$ & $19(16-20)$ & $<0.001$ \\
\hline \multicolumn{6}{|c|}{ Quality of life } \\
\hline Physical functioning (points) & $22.5(10-30)$ & $30(15-40) * * p=0.032$ & $15(5-20)$ & $17.5(10-40)$ & 0.032 \\
\hline Role physical (points) & $0(0-0)$ & $0(0-0)$ & $0(0-0)$ & $0(0-25)$ & 0.059 \\
\hline Bodily pain (points) & $36.5(22-42)$ & $41(22-41)$ & $22(22-41)$ & $31.5(22-52)$ & 0.096 \\
\hline General health (points) & $56(45-65) * * p=0.007$ & $50(30-72) * * p=0.014$ & $40(30-50)$ & $50(40-62.5)$ & 0.007 \\
\hline Vitality (points) & $40(40-50) * * p=0.037$ & $55(35-70) * * p<0.001$ & $30(20-40)$ & $40(30-52.5)$ & $<0.001$ \\
\hline Social functioning (points) & $50(37.5-62.5)$ & $56.3(37.5-87.5)$ & $50(25-50)$ & $50(37.5-62.5)$ & 0.093 \\
\hline Role emotional (points) & $0(0-0)$ & $0(0-33.3)$ & $0(0-0)$ & $0(0-33.3)$ & 0.480 \\
\hline Mental health (points) & $52(44-64)$ & $72(48-76)^{* *} p=0.001$ & $44(36-48)$ & $50(36-68)$ & 0.001 \\
\hline Physical component summary (points) & $30.5(28.4-33.9)$ & $34.1(30.5-36.1) * * p=0.014$ & $28.0(24.3-31.9)$ & $29.2(26.8-34.1)$ & 0.025 \\
\hline Mental component summary (points) & $33.9(27.8-41.4)$ & $40.4(32.9-43.8) * * p=0.005$ & $30.9(26.9-35.9)$ & $33.7(27.9-41.5)$ & 0.008 \\
\hline \multicolumn{6}{|c|}{ Histopathology } \\
\hline Mankin score (points) & $8.5(6-10) * * p=0.001$ & $8(6-8) * * p=0.035$ & $6(4.5-6.5)$ & $7(6-7)$ & 0.022 \\
\hline OARSI scale (grade) & $4(3-4)$ & $3.5(3-4)$ & $2.5(2-3)$ & $4(3-4)$ & 0.126 \\
\hline Krenn score (points) & $5(3-8)$ & $3(3-5)$ & $4(3-5)$ & $3(1-4) * p=0.049$ & 0.049 \\
\hline Average cartilage thickness, $\mathrm{mcm}$ & $6700(6200-8450)$ & $6000(4500-8600)$ & $8000(6000-9000)$ & $7500(6800-8700)$ & 0.357 \\
\hline Average vascular synovial density, \% & $23.7(18.6-35.6)$ & $18.4(6.5-20.3) * * p=0.016$ & $24.6(20.7-38.2)$ & $21.3(20.2-30.4)$ & 0.022 \\
\hline
\end{tabular}

The results are presented as a median with lower and upper quartiles: Me (LQ-UQ). P-values, except the last column, are computed via Mann-Whitney Utest and reflect different levels of parameter relevance against the following comparison groups: ${ }^{*}-$ age-related OA phenotype group; ${ }^{* *}-$ metabolic OA

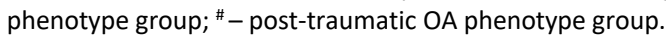




\section{Histopathological study}

After total knee replacement, surgical specimens in form of medial tibial plateau and articular synovial membrane from upper torsion of knee joint were collected and formalin-fixed in every third patient in the group (10 subjects in each group, total of 40 study participants). Histopathological study was performed at the Department of Anatomical Pathology at Orenburg State Medical University. Pyramidal sections, including cartilage and adjacent subchondral bone, as well as synovial membrane fragments, were isolated from middle one-third of the medial tibial plateau, decalcified and processed in accordance with conventional methods. The samples were sliced, and sections $(5 \mathrm{~mm})$ were stained with hematoxylin and eosin, or toluidine blue, for proteoglycan content. To access severity of OA lesions of cartilage, we used Mankin scoring system (evaluating te structure, cellular abnormalities, matrix staining and tidemark integrity) [25] and OARSI scale (assessing cartilage lesion depth) [26]. Histopathological synovitis score by V. Krenn (2002), evaluating synovial lining cell layers, density of resident cells and inflammatory infiltration [27], was used as well. Total synovitis score below 5 points was interpreted as a low-grade synovitis, while $\geq 5$ points represented a high-grade synovitis [28]. The cartilage thickness was also determined via using the eyepiece micrometer, and volumetric vascular density of synovial membrane was calculated using ocular mesh insert.

\section{Statistical analysis}

Statistical data processing was performed using Statistica 10.0 software package. To check our results for the normality of their distribution, we employed Kolmogorov-Smirnov test. For collected data, the distribution was different from normal, hence we presented our results in the form of a median with lower and upper quartiles: Me (LQ-UQ). First, we conducted nonparametric Kruskal-Wallis analysis of variance because of its applicability for comparing three or more groups. The differences were considered statistically significant at $p<0.05$. When significant differences or nearly significant differences were revealed, further pairwise detailed comparisons of phenotypes in terms of the studied parameters were carried out via nonparametric Mann-Whitney Utest (two-tailed test). To evaluate interrelations between quantitative parameters, we performed the Spearman's rankorder correlation analysis. All differences were considered significant at $p<0.05$.

\section{Results}

The results of clinical examination, quality of life assessment, and histopathological analysis are presented in Table 1.

The Kruskal-Wallis nonparametric analysis of variance showed that there were statistically significant differences between phenotypes for a number of clinical OA characteristics, histopathological indicators, and some scales of quality of life. Further pairwise comparisons of parameters revealed significant differences between particular phenotypes.

\section{Clinical data}

All subjects of age-related phenotype were significantly older than in the other groups. The highest proportion of males was in age-related OA group (male-to-female ratio $=2: 3$ ), while the lowest (2:13) was detected in metabolic and combined phenotypes. Average duration of clinical knee OA did not differ significantly among the groups. The latest OA onset was revealed in age-related OA phenotype. BMI was the key criterion for metabolic and combined phenotypes, since it obviously reflected obesity; whereas in age-related and post-traumatic OA groups, it reflected overweight subjects. In both metabolic and combined groups, patients with grade 1 obesity prevailed $(63.3 \%$ and $66.6 \%$, respectively). Similar pairwise differences (age-related vs. posttraumatic and metabolic vs. combined phenotypes) were exhibited by indicators of visceral fat analyzer and waist circumference. Hip circumference showed significant differences of age-related phenotype vs. metabolic / combined OA phenotypes.

\section{Knee joint characteristics}

We established that prevalence of Kellgren-Lawrence grade 4 was the highest among patients with combined or, less habitually, metabolic OA, e.g., in these groups, patients more often progressed to terminal radiological stage. Incidence of clinical synovitis was the highest in metabolic phenotype, while agerelated $\mathrm{OA}$ was characterized by the lowest incidence of inflammatory manifestations. Knee pain level via VAS assessment had no significant differences between the studied groups. The highest values of WOMAC index (total score) were found in metabolic $O A$, while the lowest were detected in post-traumatic phenotype. However, pain subscale WOMAC analysis revealed that metabolic and combined $O A$ were associated with higher values of knee pain, whereas stiffness levels were augmented in age-related (close to statistically significant relationship) and metabolic OA groups (significant differences vs. post-traumatic phenotype). Functional limitations were the highest in solely metabolic OA phenotype (compared with all other groups). Lequesne index score was the highest in age-related and metabolic phenotypes and had significant differences, compared with posttraumatic OA group.

\section{Quality of life in patients}

We discovered that five scales of MOS SF-36 questionnaire (physical functioning, general health, vitality and mental health) exhibited significant differences between post-traumatic and metabolic phenotypes, and two of those (general health and vitality), additionally, had significant differences between agerelated and metabolic OA groups. Physical and mental component summaries, assessing the quality of life, demonstrated the lowest (i.e., the worst) values of these parameters in patients with metabolic $\mathrm{OA}$ and the highest values in post-traumatic phenotype.

\section{Histopathological assessment}

Age-related OA phenotype was characterized by severe degenerative cartilage changes (Figure $2 \mathrm{~A}$ ) virtually over all medial tibial plateau. Deep clefts spreading into transitional and radial zones with matrix fibrillation were found; hence, cartilage was fragmented. Denuded bone formed articular surface. Sometimes synovial pannus was detected. Single chondrocyte clusters were located on edge of fissures, although, in general, hypocellularity prevailed. Tidemark was not visualized or it was breached by small vessels from subchondral bone. Toluidine blue staining revealed severe discoloration and proteoglycan loss all over noncalcified cartilage. Synovial membrane of knee joints in patients with agerelated OA was characterized by lipomatous hyperplasia (Figure 
$3 A)$, severe lymphoid and macrophage inflammatory infiltration with follicular aggregation, villous hyperplasia with cartilaginous and bone fragments inside.

In post-traumatic OA phenotype, we found cartilage thinning with deep, branched surface defects and matrix fibrillation, while bone denudation was revealed rather rarely. Chondrocytes formed giant clusters all over the cartilage (Figure 2B), in some areas it was replaced by the fibrous cartilage. Tidemark was intact or crossed by cells groups, but vascular invasion was not typical in this phenotype. Toluidine blue staining demonstrated a reduction in proteoglycan content in superficial and transitional (but rarely radial) zones. In synovial membrane (Figure 3B), synovial cell lining has been exposed to hyperplasia (4-6 layers), and moderate inflammatory infiltration was detected; in the matrix, fibrosis prevailed, and poor vascularization with blood vessel obliteration were revealed.
In cartilage of patients with metabolic OA, the cartilage surface had slight irregularities or unbranched clefts into the transitional zone, chondrocytes proliferated but did not form clusters. Key feature of this group was damaged tidemark integrity with numerous vessels penetrating noncalcified cartilage from the subchondral bone (Figure 2C). Toluidine blue staining (Figure 2D) exhibited proteoglycan content loss for $1 / 3-1 / 2$ of cartilage layer near the surface. Synovial membrane (Figure 3C) was characterized by numerous inflammatory infiltrates, at times forming follicular aggregates; also, villous hyperplasia, perivascular edema, dilated vessels with erythrocyte stasis were observed.

Combined OA phenotype had heterogeneous reorganization, combining both deep and superficial cartilage clefts, clusters of chondrocytes and hypocellularity, matrix fibrillation, tidemark breaching, and pannus formation. In synovial membrane (Figure $3 D)$, fibrosis, villous hyperplasia, and low-intensity inflammation were detected.

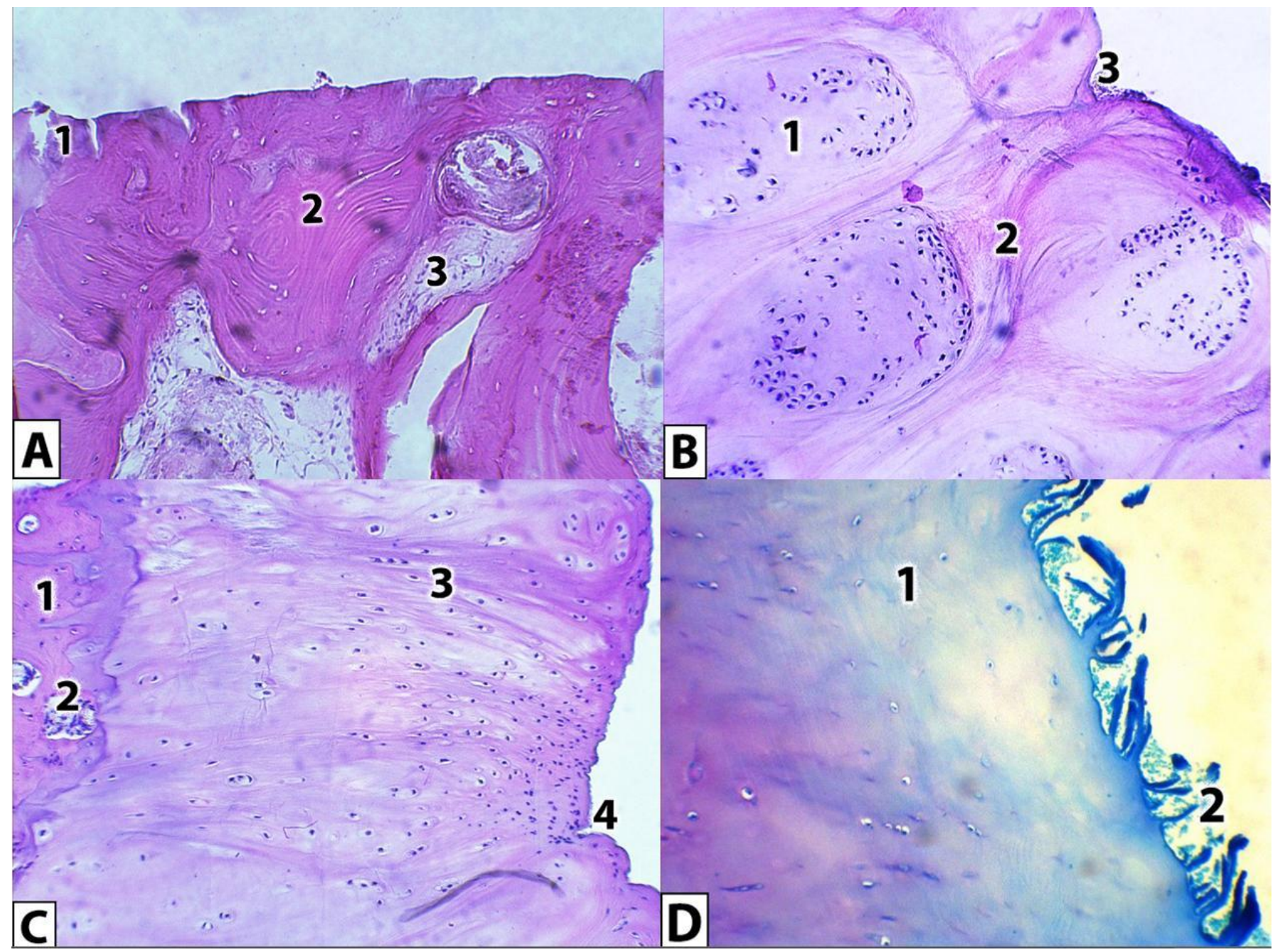

Figure 2. Histological appearances of the medial tibial plateau cartilage in patients with age-related (A), post-traumatic (B) and metabolic (C, D) OA phenotypes. Visualized with hematoxylin and eosin stain (A, B, C), or toluidine blue (D). Original magnification x150. A - Severe cartilage loss (1) with denuded bone (2), forming articular surface. Subchondral bone marrow spaces are partially replaced by fibrovascular tissue (3). B - giant chondrocyte clusters (1), surrounded by visible fibrous structures (2), were observed, minor surface clefts were present (3). C - subchondral bone hypertrophy was present in some areas (1), vascular channels breached the tidemark between calcified and non-calcified cartilage (2), chondrocytes were not subject to severe clustering, matrix fibrillation was visible (3), surface was covered by numerous clefts. D - proteoglycan content was lost at $1 / 3-1 / 2$ of the cartilage layer near the surface (1), mild loss of cartilage integrity (2) was established. 


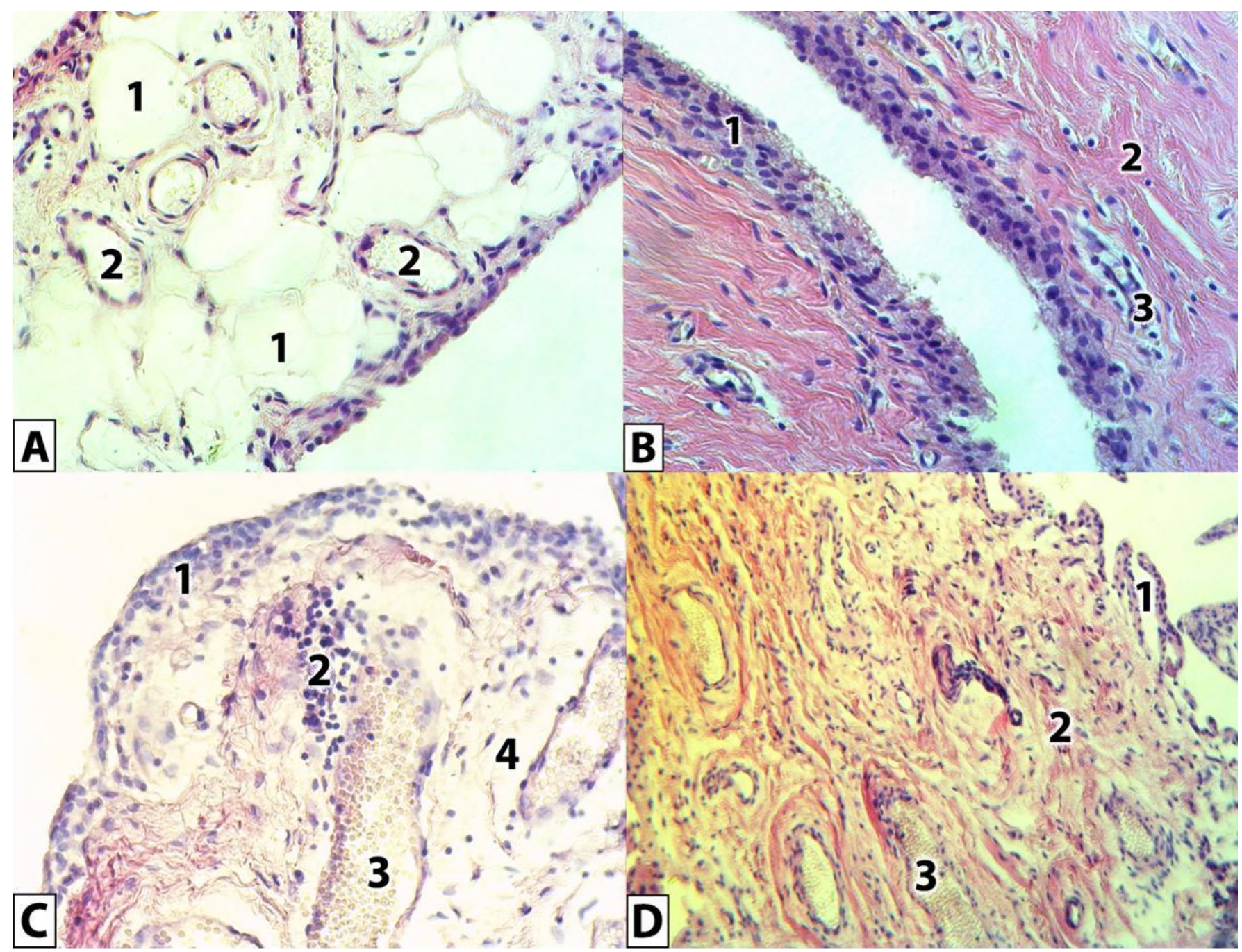

Figure 3. Histological appearances of synovial membrane of patients with age-related (A), post-traumatic (B), metabolic (C) and combined (D) OA phenotypes. Visualized with hematoxylin and eosin stain. Original magnification x300 (A, B, C), x150 (D). A - lipomatous hyperplasia of synovium, adipocytes (1), small blood vessels with mild erythrocyte stasis (2). B - synovial fibrosis with severe hyperplasia of synovial lining cell layers (1), rough thickened fibers (2), small flattened vessels (3). C - congestive and inflammatory phenomena are expressed, mild synovial lining cell hyperplasia (1), perivascular infiltration with macrophages and monocytes (2), dilated microvascular vessels with erythrocyte stasis (3), perivascular edema (4). D - small villous hyperplasia with synovial cell desquamation (1), fibrosis (2), dilated vessels with erythrocyte stasis (3).

Table 2. Coefficients of correlation between studied quantitative osteoarthritis parameters

\begin{tabular}{|c|c|c|c|c|c|c|c|c|c|c|c|c|c|}
\hline & $\begin{array}{c}\text { Patient } \\
\text { age }\end{array}$ & $\begin{array}{c}O A \\
\text { duration }\end{array}$ & $\begin{array}{c}\text { Age at } \\
\text { OA onset }\end{array}$ & $B M I$ & $\begin{array}{c}\text { Fat } \\
\text { analyzer }\end{array}$ & $\begin{array}{c}\text { Waist } \\
\text { circumference }\end{array}$ & $\begin{array}{c}\text { Hip } \\
\text { circumference }\end{array}$ & VAS & $\begin{array}{c}\text { WOMAC, } \\
\text { pain }\end{array}$ & $\begin{array}{c}\text { WOMAC, } \\
\text { stiffness }\end{array}$ & $\begin{array}{l}\text { WOMAC, } \\
\text { function }\end{array}$ & $\begin{array}{c}\text { WOMAC, } \\
\text { total }\end{array}$ & $\begin{array}{c}\text { Lequesne } \\
\text { index }\end{array}$ \\
\hline OA duration & -0.090 & & & & & & & & & & & & \\
\hline Age of OA onset & $0.637^{*}$ & -0.743 & & & & & & & & & & & \\
\hline BMI & -0.295 & 0.114 & -0.218 & & & & & & & & & & \\
\hline Fat analyzer & -0.284 & 0.203 & -0.241 & 0.696 & & & & & & & & & \\
\hline Waist circumference & -0.163 & 0.008 & -0.081 & 0.715 & 0.558 & & & & & & & & \\
\hline Hip circumference & -0.386 & 0.205 & -0.275 & 0.729 & 0.638 & 0.709 & & & & & & & \\
\hline VAS & -0.078 & 0.249 & -0.231 & 0.144 & 0.374 & -0.043 & 0.101 & & & & & & \\
\hline WOMAC, pain & -0.136 & 0.293 & -0.247 & 0.341 & 0.594 & 0.255 & 0.314 & 0.640 & & & & & \\
\hline WOMAC, stiffness & -0.063 & 0.063 & 0.020 & 0.308 & 0.497 & 0.167 & 0.227 & 0.517 & 0.646 & & & & \\
\hline WOMAC, function & -0.030 & 0.318 & -0.191 & 0.305 & 0.432 & 0.125 & 0.136 & 0.692 & 0.726 & 0.700 & & & \\
\hline WOMAC, total & -0.074 & 0.309 & -0.198 & 0.339 & 0.524 & 0.170 & 0.198 & 0.715 & 0.826 & 0.777 & 0.975 & & \\
\hline Lequesne index & 0.163 & 0.310 & -0.103 & 0.118 & 0.210 & 0.007 & 0.090 & 0.519 & 0.412 & 0.405 & 0.585 & 0.579 & \\
\hline Physical component summary & -0.005 & -0.116 & 0.094 & -0.288 & -0.430 & -0.223 & -0.072 & -0.479 & -0.471 & -0.376 & -0.652 & -0.628 & -0.616 \\
\hline Mental component summary & 0.193 & -0.046 & 0.080 & -0.304 & -0.433 & -0.405 & -0.271 & -0.497 & -0.604 & -0.699 & -0.665 & -0.714 & -0.545 \\
\hline
\end{tabular}

* significant correlations are shown in italics $(p<0.05)$. 
The comparative analysis results of histopathology of articular structures using Mankin score revealed the most pronounced cartilage lesion in age-related and post-traumatic OA groups, whereas the most preserved cartilage surface was found in metabolic phenotype. When analyzing Krenn scale for synovitis, age-related and metabolic $\mathrm{OA}$ had the highest values corresponding to high-grade synovitis, while post-traumatic and combined $O A$ had the lowest values implying the low-grade synovitis. Significant differences were found solely among agerelated vs. combined groups. Average cartilage thickness evaluation failed to reveal significant differences among the studied groups. Vascular density of synovial membrane was greater in metabolic and age-related phenotypes, which was consistent with the data on synovitis intensity, and significant differences were found between metabolic and post-traumatic OA groups with the lowest vascular densities.

\section{Correlation analysis of the results}

Table 2 demonstrates the correlation analysis results of our quantitative data.

We established that patient age correlated positively with age at $\mathrm{OA}$ onset, and negatively with obesity, that is, obesity was slightly decreasing with age, which was probably associated with age-related atrophy. Similarly, age at clinical OA onset correlated with obesity rates, and this was strong or moderate positive correlation.

When evaluating clinical and functional knee condition, we revealed significant positive correlations of WOMAC index subscales and VAS with OA duration, BMI, visceral fat content, but not patient age. It can be concluded that age per se was not associated with development of pain and functional limitations of the knee; however, OA progression (clinical duration of the disease) influenced it. Early OA onset was associated with slight increase in pain severity, since there were weak negative correlations of this indicator with VAS and WOMAC pain subscale. Among obesity indicators, fat analyzer, but not BMI, exhibited the strongest correlation with WOMAC index; it had also moderate positive correlation with VAS. When evaluating the associations of VAS, WOMAC and Lequesne index with each other, the expected significant strong and moderate positive correlations were revealed. When assessing the quality of life in patients, the correlation analysis revealed negative associations of physical and mental component summaries with obesity (BMI, fat analyzer), VAS, WOMAC scales, and Lequesne index; hence we can conclude that these factors, among all studied, had significant deteriorating effect on quality of life. Analyzing histopathological parameters, we observed significant correlation between Mankin and OARSI scores $(r=0.772)$, while Krenn scale synovitis was associated with high WOMAC value $(r=0.655)$.

\section{Discussion}

We obtained new data on clinical, functional and histopathological signs, along with quality of life, in patients with advanced knee $\mathrm{OA}$ and their associations with each other and risk factors. Depending on prevailing risk factor, distinct knee OA phenotypes, caused by age, preceding trauma, obesity, and their combination, were formed in patients with Kellgren-Lawrence grades 3-4. They were characterized by various etiology, pathways, structural tissue basis, clinical and functional manifestations, and quality of life.

Age-related OA phenotype was characterized by the latest onset, the largest average patient age, moderate to severe pain and dysfunction of knee joints sensu WOMAC and Lequesne indices, moderate deterioration in their quality of life according to MOS SF-36, and severe degenerative cartilage lesions with highgrade synovitis and lipomatous dystrophy. Forming in elderly subjects without metabolic and traumatic background with virtually $1: 1$ sex ratio, it matched the classical 'wear-and-tear' $\mathrm{OA}$ concept.

Post-traumatic OA phenotype was distinguished by the lowest pain, stiffness, functional limitations, and the highest values of mental and physical components of patient quality of life against the background of local severe cartilage lesions with reparative pattern, fibrous cartilage replacement, and synovial fibrosis with poor vascularization.

Both metabolic and combined OA phenotypes occurred more commonly in women and progressed rapidly until the terminal radiological stage (the indirect evidence of this pattern was represented by frequent occurrence of Kellgren-Lawrence grade 4). Clear metabolic OA phenotype traits were frequent clinical synovitis, the most severe knee pain, stiffness, functional limitations, the worst quality of life, vascular invasion of the cartilage with shallow surface lesion, high-grade synovitis with intensive vascularization, and villous hyperplasia.

Combined OA phenotype had diversified clinical manifestations and histopathological features, probably, because of other risk factors' impact.

A comparative analysis of different OA phenotypes indicated the presence of clinical and morphological inconsistencies. Indeed, despite the fact that the most severe structural changes were observed in patients with age-related OA phenotype, the highest values of pain, functional disorders, and the worst quality of life were found in metabolic OA group, characterized by the lowest histopathological cartilage scores. Patients with post-traumatic OA phenotype had the lowest clinical and functional disorders and satisfactory quality of life, while deep cartilage defects were revealed in the histopathological study. However, these data could not be considered conflicting (in regards to clinical vs. histopathological evidence), if vascularization of the joint structures was taken into account as a pain-associated pathway (which was confirmed by the positive correlation of Krenn scale with WOMAC index). In fact, both synovial vascularization activation and severe cartilage vessels' invasion were found in metabolic OA phenotype, even though cartilage surface was damaged insignificantly, so that the group had the most unfavorable clinical course. Similarly, post-traumatic OA phenotype with synovial fibrosis and poor vascular density (and no vessel penetration into the cartilage) had the lowest pain level.

Hence, we conclude that presence of clinical and morphological differences between the phenotypes undoubtedly requires individualized approach to treatment of different categories of $O A$ patients. It is not a proper strategy to use generalized management for some 'average' OA, the prototype for which was the combined phenotype. As age-related OA had severe cartilage lesion up to its full absence with bone denudation, it could be assumed that prescribing symptomatic slow acting drugs for OA (SYSADOA) would not be very effective in this group, since the substrate (articular cartilage) was practically absent. 
Furthermore, high-grade histopathological synovitis requires use of nonsteroidal anti-inflammatory drugs (NSAIDs), taking into account the risk/benefit in relation to comorbid diseases, because elderly population is characterized by higher comorbidity. Some studies recommend avoiding NSAIDs, whenever possible, in patients with cardiovascular diseases, or else choosing the shortest duration and the lowest effective doses [29]. In treatment of post-traumatic phenotype, it is necessary to stimulate metabolism and reparative capacity of cartilage tissues. This statement is supported by our finding of regenerative potencies in patients against the background of deep uneven cartilage damage. The metabolic OA is the most difficult phenotype in terms of treatment, because of severe clinical manifestations and comorbid obesity/metabolic disorders, which, according to the literature, are associated with enlarged risk of knee replacement [30]. Perhaps, pain syndrome in this group is more influenced not by cartilage damage per se, but by subchondral bone lesion and vascular invasion, since all patients exhibited these features in histopathological study. Frequent clinical and morphologically verified synovitis determines the need for high doses and long courses of NSAIDs. Correction of microvascular disorders has the potential to significantly improve clinical $O A$ course as well. Treatment of metabolic OA should be comprehensive, including control of risk factors, such as obesity (weight normalization) and metabolic syndrome.

It is interesting to consider our study in the context of other proposed OA phenotypes. Herrero-Beaumont et al. (2017) [15] proposed the existence of four clinical phenotypes: biomechanical, osteoporotic, metabolic and inflammatory. Although there were no clear criteria of correspondence to phenotypes, we hypothesize that the biomechanical phenotype corresponds predominantly to post-traumatic OA in our study, because it presumes, "...mechanical stress as major factors involved in the development and progression of $O A$, particularly in weight-bearing joint," and our patients of post-traumatic OA group had confirmed trauma as a key risk factor for knee OA. Then, in the mentioned article, osteoporotic phenotype was considered in menopausal women, and the association with estrogen deficiency was noted. Our agerelated phenotype corresponds to this phenotype only partially, because it includes older men in addition to menopausal women and suggests not so much the relationship with estrogen as emphasizes the lack of other risk factors. Metabolic OA is only phenotype with sufficiently clear criteria (obesity, metabolic syndrome). As for inflammatory phenotype, in our opinion, it is controversial, because most patients in our study from agerelated, post-traumatic and metabolic OA groups had inflammatory signs including histopathological and clinical synovitis. Probably, the authors of the above classification meant highly active $\mathrm{OA}$, similar to rheumatoid arthritis, which was not represented in our study. Thus, our study complements current understanding of OA phenotypes and is consistent with the latest data on their classification.

In addition to comparative analysis of OA phenotypes, we evaluated relationships between clinical, functional, histopathological, and other features. When investigating sex ratios, female prevalence was detected, which was consistent with conventional published data on knee joint, stating that knee $\mathrm{OA}$ affected women more often than men [31]. Also, it was confirmed that in older age groups with knee OA (closer to 80 years old), the sex ratio is approaching 1:1 [31].
Obesity and early clinical OA onset were associated with severe pain and other unfavorable manifestations of knee $O A$, identified by WOMAC, Lequesne index and VAS. WOMAC index was more informative for knee condition assessment, compared with VAS and Lequesne index. Also, in our study, obesity and metabolic disorders significantly aggravated the course of knee $\mathrm{OA}$, which was consistent with relevant data of most publications $[32,33,34]$. Remarkably, visceral fat content was a better obesity indicator then BMI.

Increasing pain, stiffness, and functional disorders of knees, as well as obesity (according to BMI, fat analyzer), worsened physical and mental component summaries of quality of life in patients. Histopathological synovitis severity was associated with more intensive pain and functional limitations in patients at late-stage knee OA, which was consistent with the data reported in [35].

\section{Conclusion}

We conducted the first ever comprehensive comparative analysis of physical examination data, clinical and functional parameters, knee joint tissue morphology, and quality of life in patients with osteoarthritis of various genesis: age-related, posttraumatic, metabolic and combined. The methodological basis for differential approach to treating different categories of osteoarthritis patients was developed.

\section{Ethical approva}

All procedures performed in studies involving human participants were in accordance with the ethical standards of the institutional and/or national research committee and with 1964 Declaration of Helsinki and its later amendments or comparable ethical standards.

\section{Conflict of interest}

We declare no conflicts of interest.

\section{References}

1. Kraus VB, Blanco FJ, Englund M, Karsdal MA, Lohmander LS. Call for standardized definitions of osteoarthritis and risk stratification for clinical trials and clinical use. Osteoarthritis Cartilage 2015; 23(8): 1233-1241. https://doi.org/10.1016/j.joca.2015.03.036.

2. Wallace IJ, Worthington S, Felson DT, Jurmain RD, Wren KT, Maijanen $\mathrm{H}$, et al. Knee osteoarthritis has doubled in prevalence since the mid20th century. Proc Natl Acad Sci U S A 2017; 114(35): 9332-9336. https://doi.org/10.1073/pnas.1703856114.

3. Smith L, Wittenauer R, Aden K, Duthey B. Priority diseases and reasons for inclusion. Osteoarthritis. In: Kaplan W, Wirtz V, Mantel A, Stolk P, Duthey B, Laing R, Eds. Priority Medicines for Europe and the World 2013 Update. Geneva, Switzerland: World Health Organisation. 2013: 126-128.

https://www.who.int/medicines/areas/priority medicines/MasterDoc June28 FINAL Web.pdf.

4. Bruyère $\mathrm{O}$, Cooper $\mathrm{C}$, Arden $\mathrm{N}$, Branco J, Brandi ML, Herrero-Beaumont $\mathrm{G}$, et al. Can we identify patients with high risk of osteoarthritis progression who will respond to treatment? A focus on epidemiology and phenotype of osteoarthritis. Drugs Aging 2015; 32(3): 179-187. https://doi.org/10.1007/s40266-015-0243-3.

5. Deveza LA, Nelson AE, Loeser RF. Phenotypes of osteoarthritis: Current state and future implications. Clin Exp Rheumatol 2019; 37 Suppl 120(5): 64-72. https://pubmed.ncbi.nlm.nih.gov/31621574/.

6. Van Spil WE, Kubassova O, Boesen M, Bay-Jensen AC, Mobasheri A Osteoarthritis phenotypes and novel therapeutic targets. Biochem 
Pharmacol 2019; 165: 41-48. https://doi.org/10.1016/j.bcp.2019.02.037.

7. Mobasheri A, Saarakkala S, Finnilä M, Karsdal MA, Bay-Jensen AC, van Spil WE. Recent advances in understanding the phenotypes of osteoarthritis. F1000Res 2019; 8: F1000 Faculty Rev-2091. https://doi.org/10.12688/f1000research.20575.1.

8. Jeremiasse B, Welsing PMJ, Fellows C, Lafeber FP, Van Spil WE. Identification of biochemical phenotypes in knee osteoarthritis: Longitudinal data from the FNIH OA biomarker consortium. Ann $\begin{array}{llll}\text { Rheum Dis 2017; 2): } 964 . & \text { 76(Suppl }\end{array}$ https://doi.org/10.1136/annrheumdis-2017-eular.5825.

9. Carlson AK, Rawle RA, Wallace CW, Brooks EG, Adams E, Greenwood $\mathrm{MC}$, et al. Characterization of synovial fluid metabolomic phenotypes of cartilage morphological changes associated with osteoarthritis. Osteoarthritis Cartilage 2019; 27(8): 1174-1184. https://doi.org/10.1016/j.joca.2019.04.007.

10. Waarsing JH, Bierma-Zeinstra SM, Weinans $\mathrm{H}$. Distinct subtypes of knee osteoarthritis: Data from the Osteoarthritis Initiative. Rheumatology (Oxford) 2015; 54(9): 1650-1658. https://doi.org/10.1093/rheumatology/kev100.

11. Radojčić MR, Arden NK, Yang X, Strauss VY, Birrell F, Cooper C, et al; VIDEO trial investigators. Pain trajectory defines knee osteoarthritis subgroups: A prospective observational study. Pain 2020; 161(12): 2841-2851. https://doi.org/10.1097/j.pain.0000000000001975.

12. Heard BJ, Fritzler MJ, Wiley JP, McAllister J, Martin L, El-Gabalawy H, et al. Intraarticular and systemic inflammatory profiles may identify patients with osteoarthritis. J Rheumatol 2013; 40(8): 1379-1387. https://doi.org/10.3899/irheum.121204.

13. Lila AM, Alekseeva LI, Telyshev KA. Current approaches to osteoarthritis phenotyping. Modern Rheumatology Journal 2019; 13(2): 4-8. Russian. https://doi.org/10.14412/1996-7012-2019-2-4-8.

14. Bruyère $\mathrm{O}$, Honvo $\mathrm{G}$, Veronese $\mathrm{N}$, Arden NK, Branco J, Curtis EM, et al. An updated algorithm recommendation for the management of knee osteoarthritis from the European Society for Clinical and Economic Aspects of Osteoporosis, Osteoarthritis and Musculoskeletal Diseases (ESCEO). Semin Arthritis Rheum 2019; 49(3): 337-350. https://doi.org/10.1016/j.semarthrit.2019.04.008.

15. Herrero-Beaumont $G$, Roman-Blas JA, Bruyère $O$, Cooper $C$, Kanis J, Maggi S, et al. Clinical settings in knee osteoarthritis: Pathophysiology guides treatment. Maturitas 2017; 96: 54-57 https://doi.org/10.1016/j.maturitas.2016.11.013.

16. van Spil WE, Bierma-Zeinstra SMA, Deveza LA, Arden NK, Bay-Jensen $A C$, Kraus VB, et al. A consensus-based framework for conducting and reporting osteoarthritis phenotype research. Arthritis Res Ther 2020; 22(1): 54. https://doi.org/10.1186/s13075-020-2143-0.

17. Deveza LA, Melo L, Yamato TP, Mills K, Ravi V, Hunter DJ. Knee osteoarthritis phenotypes and their relevance for outcomes: A systematic review. Osteoarthritis Cartilage 2017; 25(12): 1926-1941. https://doi.org/10.1016/i.joca.2017.08.009.

18. Zaki S, Smith MM, Smith SM, Little CB. Differential patterns of pathology in and interaction between joint tissues in long-term osteoarthritis with different initiating causes: Phenotype matters. Osteoarthritis Cartilage 2020; 28(7): 953-965. https://doi.org/10.1016/j.joca.2020.04.009.

19. Berenbaum F. Deep phenotyping of osteoarthritis: A step forward. Ann Rheum Dis 2019; 78(1): 3-5. https://doi.org/10.1136/annrheumdis2018-213864.

20. Altman RD. Criteria for classification of clinical osteoarthritis. J Rheumatol Suppl 1991; 27: 10-12. https://pubmed.ncbi.nlm.nih.gov/2027107.

21. Kellgren JH, Lawrence JS. Radiological assessment of osteoarthrosis. $\begin{array}{lllll}\text { Ann } & \text { Rheum 16(4): } & \text { 494-502. }\end{array}$ https://doi.org/10.1136/ard.16.4.494.

22. Bellamy N, Buchanan WW, Goldsmith $\mathrm{CH}$, Campbell J, Stitt LW. Validation study of WOMAC: A health status instrument for measuring clinically important patient relevant outcomes to antirheumatic drug therapy in patients with osteoarthritis of the hip or knee. J Rheumato 1988; 15(12): 1833-1840. https://pubmed.ncbi.nlm.nih.gov/3068365.

23. Lequesne MG, Mery C, Samson M, Gerard P. Indexes of severity for osteoarthritis of the hip and knee. Validation - value in comparison with other assessment tests. Scand J Rheumatol Supp/ 1987; 65: 85-89. Erratum in: Scand J Rheumatol 1988; 17(3): following 241. Erratum in Scand J Rheumatol Suppl 1988; 73: 1. https://doi.org/10.3109/03009748709102182.

24. Ware JE, Kosinski M, Keller S. SF-36 physical and mental health summary scales: A user's manual. Boston, MA: Health Assessment Lab. 1994; 190 p. https://www.researchgate.net/profile/John-Ware6/publication/292390260_SF-

36 Physical and Mental Health Summary Scales a User\%27s Man ual/links/5af580264585157136caee31/SF-36-Physical-and-MentalHealth-Summary-Scales-a-Users-Manual.pdf.

25. Mankin HJ. Biochemical and metabolic aspects of osteoarthritis. Orthop Clin North Am 1971; 2(1): 19-31. https://pubmed.ncbi.nlm.nih.gov/4940528.

26. Kraus VB, Huebner JL, DeGroot J, Bendele A. The OARSI histopathology initiative - recommendations for histological assessments of osteoarthritis in the guinea pig. Osteoarthritis Cartilage 2010; 18 Suppl 3(Suppl 3): S35-S52. https://doi.org/10.1016/j.joca.2010.04.015.

27. Krenn V, Morawietz L, Häupl T, Neidel J, Petersen I, König A. Grading of chronic synovitis - a histopathological grading system for molecular and diagnostic pathology. Pathol Res Pract 2002; 198(5): 317-325. https://doi.org/10.1078/0344-0338-5710261.

28. Krenn V, Morawietz L, Burmester GR, Kinne RW, Mueller-Ladner U, Muller B, Haupl T. Synovitis score: Discrimination between chronic low-grade and high-grade synovitis. Histopathology 2006; 49(4): 358364. https://doi.org/10.1111/j.1365-2559.2006.02508.x.

29. Turkiewicz A, Gerhardsson de Verdier M, Engström G, Nilsson PM, Mellström C, Lohmander LS, et al. Prevalence of knee pain and knee $\mathrm{OA}$ in southern Sweden and the proportion that seeks medical care. Rheumatology (Oxford) 2015; 54(5): 827-835. https://doi.org/10.1093/rheumatology/keu409.

30. Cherkasskiy L, Caffrey JP, Szewczyk AF, Cory E, Bomar JD, Farnsworth $\mathrm{CL}$, et al. Patient-specific 3D models aid planning for triplane proximal femoral osteotomy in slipped capital femoral epiphysis. J Child Orthop 2017; 11(2): 147-153. https://doi.org/10.1302/1863-2548-11-170277.

31. Oliveria SA, Felson DT, Reed JI, Cirillo PA, Walker AM. Incidence of symptomatic hand, hip, and knee osteoarthritis among patients in a health maintenance organization. Arthritis Rheum 1995; 38(8): 1134 1141. https://doi.org/10.1002/art.1780380817.

32. Lee KM, Chung CY, Sung KH, Lee SY, Won SH, Kim TG, et al. Risk factors for osteoarthritis and contributing factors to current arthritic pain in South Korean older adults. Yonsei Med J 2015; 56(1): 124-131. Erratum in: Yonsei Med J 2015; 56(2): 591. Erratum in: Yonsei Med J 2016; 57(3): 806. https://doi.org/10.3349/ymj.2015.56.1.124.

33. Baudart P, Louati K, Marcelli C, Berenbaum F, Sellam J. Association between osteoarthritis and dyslipidaemia: A systematic literature review and meta-analysis. RMD Open 2017; 3(2): e000442. https://doi.org/10.1136/rmdopen-2017-000442.

34. Courties A, Berenbaum F, Sellam J. The phenotypic approach to osteoarthritis: A look at metabolic syndrome-associated osteoarthritis. Joint Bone Spine 2019; 86(6): 725-730. https://doi.org/10.1016/j.jbspin.2018.12.005.

35. Wyatt LA, Moreton BJ, Mapp PI, Wilson D, Hill R, Ferguson E, et al. Histopathological subgroups in knee osteoarthritis. Osteoarthritis Cartilage 2017; 25(1): 14-22. https://doi.org/10.1016/j.joca.2016.09.021.

\section{Authors:}

Kristina V. Korochina - MD, PhD, Assistant, Department of Internal Diseases, Orenburg State Medical University, Orenburg, Russia. https://orcid.org/0000-0003-2110-1428. 
Tatyana V. Chernysheva - MD, DSc, Head of Department of Internal Diseases, Orenburg State Medical University, Orenburg, Russia. https://orcid.org/0000-0001-9645-5816.

Irina E. Korochina - MD, PhD, Assistant Professor, Department of Outpatient Therapy, Orenburg State Medical University, Orenburg, Russia. https://orcid.org/0000-0001-9956-4508. 